\title{
Evaluation of Respiratory Therapist Extender Comfort With Mechanical Ventilation During COVID-19 Pandemic
}

\author{
Karsten J Roberts, Bridgette Johnson, Heather M Morgan, Jody M Vrontisis, Katie M Young, \\ Edward Czerpak, Barry D Fuchs, and Margie Pierce
}

\begin{abstract}
BACKGROUND: Staffing strategies used to meet the needs of respiratory care departments during the COVID-19 pandemic included the deployment of respiratory therapist extenders. The purpose of this study was to evaluate respiratory therapist extenders' comfort level with critical care ventilators while caring for patients with COVID-19. To our knowledge, this is the first study to evaluate the deployment of certified registered nurse anesthetists (CRNAs) in a critical care setting. METHODS: A qualitative survey method was used to assess CRNA experience with critical care ventilators. Prior to deployment in the ICU, CRNAs were trained by clinical lead respiratory therapists. Education included respiratory clinical practices and ventilator management. Sixtyminute sessions were held with demonstration stations set up in ICUs for hands-on experience. RESULTS: Fifty-six CRNAs responded to our survey (63\%). A mean \pm SD of $9.48 \pm 12.27 \mathrm{~h}$ was spent training prior to deployment in the ICU. CRNAs were at the bedside a mean \pm SD of 73.0 \pm 40.6 $\mathrm{h}$ during the pandemic. While CRNA comfort level with critical care ventilators increased significantly $(P<.001)$ from the beginning to the end of their work experience, no statistically significant differences were found between CRNA comfort based on years of experience. Differences in comfort level were not found after training (chi-squared test $23.82, P=.09$ ) or after ICU experience was completed (chi-squared test $=15.99, P=.45$ ). Similarly, mean comfort level did not increase based on the number of hours spent working in the ICU (chi-squared test $=13.67$, $P=.55)$. CONCLUSIONS: Comfort level with mechanical ventilation increased for CRNAs working alongside respiratory therapists during the COVID-19 pandemic. Key words: advanced practice nursing; critical care; interdisciplinary health team; pandemics; mechanical ventilators; respiratory therapy. [Respir Care 2021;66(2):199-204. (C) 2021 Daedalus Enterprises]
\end{abstract}

\section{Introduction}

Patients with severe COVID-19 infection may develop unique lung injury and may require expertly managed mechanical ventilation. ${ }^{1,2}$ Many of the required interventions are carried out by respiratory therapists (RTs), the experts

Mr Roberts, Ms Johnson, Ms Morgan, Ms Vrontisis, Ms Young, Dr Fuchs, and Ms Pierce are affiliated with the Department of Respiratory Care, Hospital of the University of Pennsylvania, Philadelphia, Pennsylvania. Mr Czerpak is affiliated with the Department of Anesthesia - CRNAs, University of Pennsylvania Health System, Philadelphia, Pennsylvania. Dr Fuchs is affiliated with the Division of Pulmonary, Allergy, and Critical Care, Department of Medicine, University of Pennsylvania, Philadelphia, Pennsylvania.

Supplementary material related to this paper is available at http://www. rcjournal.com. on the application of mechanical ventilation. ${ }^{3}$ COVID-19 presents a significant strain on health care resources, such as the availability of qualified RTs, due to the sudden and quick spread within geographic regions. ${ }^{4-6}$

Rapid and efficient decisions are required to prepare for patients requiring mechanical ventilation. ${ }^{7-10}$ Leaders must have a plan and rapidly deploy these strategies during a crisis. ${ }^{7,8,11}$ Strategies used during pandemics include stockpiling

\footnotetext{
The authors have disclosed no conflicts of interest.

Correspondence: Karsten J Roberts MSc RRT, Department of Respiratory Care Services, Hospital of the University of Pennsylvania, 3400 Spruce St, Ground Floor, Founders Building, Philadelphia, PA 19104. E-mail:karsten.j.roberts@gmail.com.
}

DOI: $10.4187 /$ respcare. 08459 


\section{RT Extenders' Comfort With Mechanical Ventilation}

equipment, active supply chain management, opening of novel ICU space, and training RTs on new equipment (eg, helmet CPAP and home care ventilators). ${ }^{710}$ Because respiratory care services are in high demand, staffing strategies

\section{See the Related Editorial on Page 351}

focused on increasing the number of RTs are recommended to include non-critical care health professionals. ${ }^{7,9,10}$ To meet these needs, other professionals, including medical students and certified registered nurse anesthetists (CRNAs), were trained to function as respiratory therapy extenders (RTEs) during the COVID-19 pandemic. ${ }^{9}$

Nurse anesthetists are advanced practice nurses who collaborate with a variety of specialties and provide a valuable resource to hospitals, including the basic knowledge of mechanical ventilation and airway management. ${ }^{11,12}$ In our health system, CRNAs were made available to assist respiratory care services due to the elimination of elective procedures. The Commonwealth of Pennsylvania Health Department recommended strategies for interdisciplinary collaboration to overcome staffing and other resource issues during the COVID-19 pandemic. ${ }^{13}$ Recognizing the potential for pandemics and other mass casualty events, the American Association for Nurse Anesthetists provided guidelines for the role of CNRAs in crisis management, with 2 areas of focus that included preparedness and response. ${ }^{11,14}$ In consideration of these factors, CRNAs were chosen to collaborate with the respiratory care department as RTEs.

The purpose of this study was to evaluate the comfort level with critical care ventilators experienced by RTEs while caring for patients during the COVID-19 pandemic. To our knowledge, this is the first study to evaluate the deployment of CRNAs as RTEs in a critical care setting.

\section{Methods}

We utilized a just-in-time, hands-on training model to educate CRNAs at the Hospital of the University of Pennsylvania and Penn Presbyterian Medical Center, Philadelphia, PA. Education sessions were led by clinical lead RTs at demonstration stations in the ICU. Training sessions lasted about 60 min and included respiratory clinical practices and ventilator management. After each session, CRNAs were given the opportunity to practice at each station for several hours throughout the day. CRNAs were guided through the use of 4 models of critical care ventilators used in our institutions. In addition to learning about ventilator interfaces, CRNAs were educated on various modes of ventilation used in the critical care setting. Training sessions were held over the course of 1 week from April 8, 2020, to April 15, 2020.

CRNAs were deployed in the ICUs from April 14, 2020, to May 16, 2020. During the first week of deployment,

\section{QUICK LOOK}

\section{Current knowledge}

During times of high patient volume, respiratory care resources may be stretched beyond capacity. Other health care professionals may be called upon to assist respiratory therapists' management of mechanical ventilation during times of crisis. Mechanical ventilation requires expert oversight for safe operation.

\section{What this paper contributes to our knowledge}

Certified Registered Nurse Anesthetists (CRNAs) were deployed to support respiratory therapists during the COVID-19 pandemic. This strategy was executed safely with no reports of adverse events related to mechanical ventilation. CRNAs comfort with critical care ventilators increased as a result of working closely with respiratory therapists.

CRNAs were scheduled for $8-10 \mathrm{~h}$ of shadowing the respiratory care workflow in the ICUs. During this time, CRNAs had the opportunity to get experience with charting patient-ventilator assessments. They received additional training on transport ventilators that would be used in surge ICUs should the demand for ventilators exceed the availability of critical care ventilators. CRNAs also observed respiratory care interactions during critical care physician rounds. Once shadowing was completed, CRNAs were assigned to an ICU to work with a RT for a 12-h shift. Coverage was provided $24 \mathrm{~h} / \mathrm{d}$, during which CRNAs assisted with ventilator management, including assessment of patient-ventilator interaction and charting. Other responsibilities included critical care procedures, respiratory therapies, and care planning.

After the initial patient surge, we used a qualitative survey method to assess CRNA experiences with critical care ventilators (see the supplementary materials at http://www. rcjournal.com). The 16-question survey included demographics, questions related to training, ICU experience during COVID-19, and overall experience. To define overall comfort with ventilators, we used a Likert scale (1-10), with 1 being the least comfortable and 10 being the most comfortable. Two open-ended questions asked the CRNA to describe the most positive aspect of their experience and any concerns. Study data were collected and managed using REDCap (Research Electronic Data Capture, Vanderbilt University, Nashville, Tennessee), secured through the University of Pennsylvania. After the study was determined to be exempt by the University of Pennsylvania Institutional Review Board, the survey was distributed to the CRNAs at 2 institutions within our health system in June 2020. The survey remained 


\section{RT Extenders' Comfort With Mechanical Ventilation}

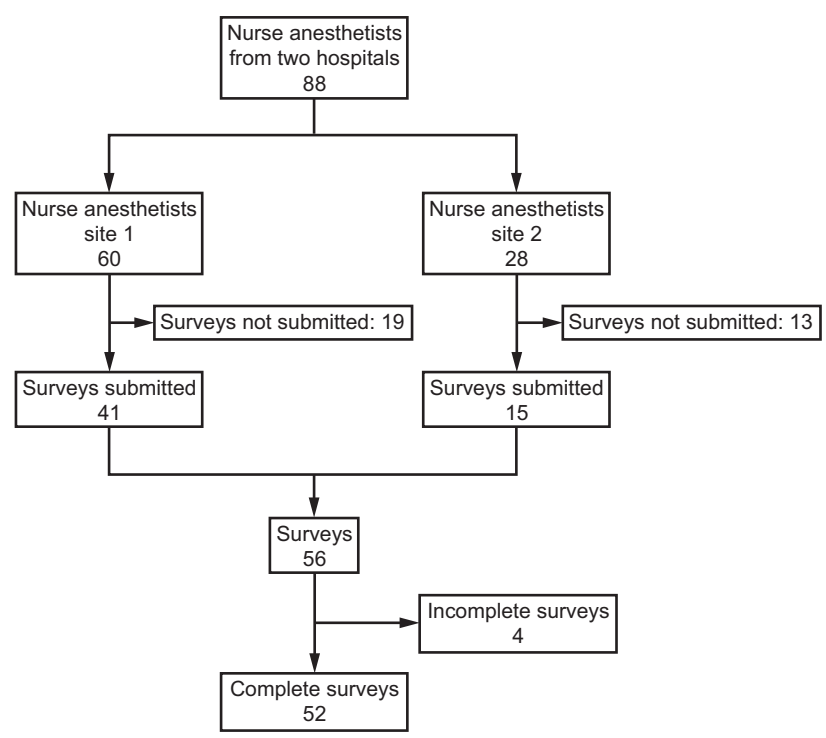

Fig. 1. Flow chart.

open for 2 weeks with 1 reminder email sent halfway through the survey period.

\section{Statistical Analysis}

Statistical analysis was completed using SPSS Statistics for Mac 26 (IBM, Armonk, New York). Descriptive statistics were reported in means and standard deviations. Categorical data were reported as percentages. Ordinal responses were considered non-parametric. Friedman's test was used to determine differences between groups, and post hoc pairwise comparisons were made using the Wilcoxon signed-ranks test. A Kruskal-Wallis test was used to compare nonparametric, independent samples. For all statistical tests, $P<.05$ was considered significant.

\section{Results}

The survey was sent to 88 CRNAs across 2 hospitals in our health system. We had an overall response rate of $63.3 \%(n=56)$. Four of the surveys received were excluded due to incompleteness. A total of 52 surveys were included in data analysis (Fig. 1). All of the respondents had at minimum a master's degree, while 5 respondents reported having doctoral degrees. The mean \pm SD years of experience working as a CRNA was $7.43 \pm 6.35$ (Table 1). The mean \pm SD number of hours CRNAs reported training with critical care ventilators prior to working in the ICU was $9.48 \pm 12.27$. The mean \pm SD number of hours the CRNAs were at the bedside during the pandemic was $72.96 \pm 40.59$.

CRNAs were asked to report comfort level with critical care ventilators prior to the pandemic, after education with RT staff, and after their ICU experience was completed.
Table 1. Demographics

\begin{tabular}{lc}
\hline \hline Certified registered nurse anesthetists & \\
Hospital site 1 & $38(73)$ \\
Hospital site 2 & $14(27)$ \\
Highest education & \\
Master's degree & $26(50)$ \\
Second master's degree & $20(38)$ \\
Master's of business administration & $1(2)$ \\
Doctoral degree & $5(10)$ \\
Years of experience & $7.43 \pm 6.35$ \\
Hours spent training & $9.48 \pm 12.27$ \\
Hours working with critical care ventilator & $72.96 \pm 40.59$
\end{tabular}

Data are presented as $n(\%)$ or mean \pm SD. $N=52$ respondents

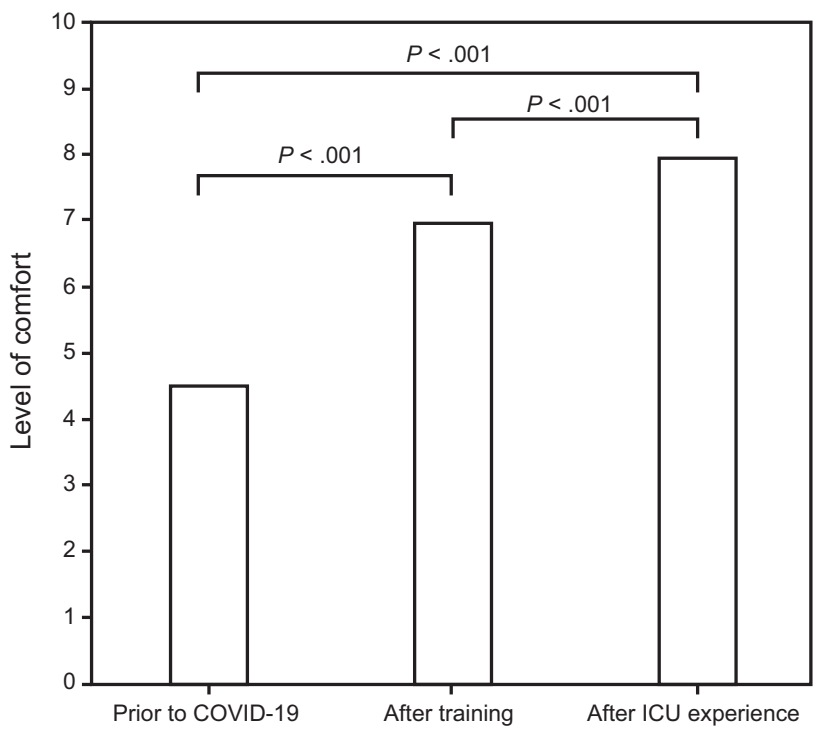

Fig. 2. Wilcoxon signed ranks test comparing mean levels of comfort with critical care ventilators.

Mean \pm SD comfort levels with ventilators were $4.52 \pm$ 2.06 prior to the pandemic, $6.08 \pm 1.89$ after training with RTs, and $7.79 \pm 1.55$ after ICU experience. Comfort level with ventilators increased significantly at these time markers $(P<.001)$ (Fig. 2). No statistical differences were found between CRNA comfort level with critical care ventilators based on years of experience (Table 2). No difference was found in mean comfort when measured after training (chi-square $=23.82, P=.09$ ) or after ICU experience was completed (chi-square $=15.99, P=.45$ ). Similarly, mean comfort level did not increase based on the number of hours spent working in the ICU (chi-square = $13.67, P=.55)$.

Mean \pm SD satisfaction was reported as $6.65 \pm 2.46$ for overall experience of working as RTE. Respondents also indicated they received an appropriate amount of training during orientation to the RTE role $(5.23 \pm 2.25)$. Although 


\section{RT Extenders' Comfort With Mechanical Ventilation}

Table 2. Comparison of CRNA-Reported Comfort With Critical Care Ventilator and Years of Experience

\begin{tabular}{lccr}
\hline \hline & Comfort Level & Chi-Square & $P$ \\
\hline Prior to COVID-19 & $4.52 \pm 2.06$ & 14.69 & .68 \\
After training with RRT & $6.98 \pm 1.89$ & 18.47 & .43 \\
After working at bedside & $7.79 \pm 1.55$ & 24.14 & .15 \\
& & & \\
Comfort level presented as mean \pm SD. Comparison performed with the Kruskal-Wallis test. \\
Statistical significance set to $P<.05$. \\
CRNA = certified registered nurse anesthetist \\
RRT = registered respiratory therapist \\
\end{tabular}

Table 3. Overall Experience and Feedback

\begin{tabular}{lc}
\hline \hline Experience (Likert scale 1-10) & \\
Satisfaction with experience & $6.65 \pm 2.46$ \\
Reliance on RT during work & $7.46 \pm 1.89$ \\
CRNA added value & $7.60 \pm 2.25$ \\
$\quad$ More education needed & $4.77 \pm 2.25$ \\
Positive feedback* & \\
Interdisciplinary collaboration & $20(54)$ \\
Professionalism & $16(43)$ \\
Skills used & $11(30)$ \\
Teamwork & $9(24)$ \\
Patient care/safety & $4(11)$ \\
$\quad$ Understanding COVID-19 & $2(0.5)$ \\
Constructive feedback $\dagger$ & \\
$\quad$ More education needed & $10(66)$ \\
CRNA ineffective as RRT & $9(60)$ \\
Concern for charting & $4(27)$ \\
Lack of professionalism & $2(13)$ \\
Overwhelmed & $2(1)$ \\
Concern for next surge & $2(1)$ \\
\hline Data are presented as mean \pm SD or $n(\%)$. & \\
$* n=37$ & \\
${ }^{\dagger} n=15$ & \\
RT respiratory therapist & \\
RRT $=$ registered respiratory therapist & \\
\hline
\end{tabular}

CRNAs reported relying on the RT for help at the bedside (7.46 \pm 1.89$)$, CRNAs did consider their role as RTEs an added value in patient care $(7.60 \pm 2.25)$ (Table 3$)$.

Open-ended questions related to overall experience included both positive $(n=37)$ and constructive $(n=15)$ feedback. Comments related to interprofessional collaboration were mentioned most often $(n=20)$. Forty-three percent of the positive comments expressed gratitude for the professionalism displayed during the pandemic. CRNAs were also pleased to have their skills called upon during the pandemic and appreciated the teamwork. Concerns were expressed by $29 \%$ of the respondents to our survey. Of the constructive feedback received, $66 \%$ of respondents felt that more training would be beneficial. Correspondingly, 9 of the anesthetists stated they were inadequate to replace
RTs at the bedside. Concerns over charting, lack of professionalism, feeling overwhelmed, and concern regarding future needs were also expressed in the comments.

\section{Discussion}

In this study, our results indicate that overall comfort level with using critical care ventilators increased over time among CRNAs working in the ICU during the COVID-19 pandemic. CRNAs typically come from a critical care background, with a minimum of $2 \mathrm{y}$ of nursing experience required for admission to CRNA programs. ${ }^{14}$ Although no statistically significant differences were found based on years of experience, the traditional role of CRNAs in the operating room lends itself to some familiarity with the basics of mechanical ventilation. The role of a CRNA is adaptable, resilient, and expert when managing airways and resuscitating patients, not only in the operating room but during clinical emergencies. ${ }^{11,14}$

Nurse anesthetists are advanced practice registered nurses who are committed to excellence, patient safety, and providing services throughout the hospital at a reasonable cost to health care systems. ${ }^{14}$ Interdisciplinary collaboration is inherent in the CRNA role because they work primarily in the operating room and collaborate with surgeons, physician anesthesiologists, and other qualified health care professionals. ${ }^{11,12,14}$ CRNAs have acquired all the skills required to provide specific care and technical procedures in the fields of intensive care anesthesia, which allows them to care for and manage COVID-19 patients effectively. ${ }^{14}$ The aforementioned qualities and their role in the hospital made CRNAs a suitable fit both as RTEs and for inclusion in this study.

Comfort level with mechanical ventilation did not increase significantly based on the amount of time spent training. This may be explained in a number of ways. Comments from our survey included the perceived need for more education prior to deployment. We acknowledge that rapid deployment, on short notice, could lead to some level of unease related to the technical aspects of respiratory care. However, just-in-time education is the standard for quickly deploying support staff during medical crises. ${ }^{7,10,11}$ The recommendations from the Commonwealth of Pennsylvania Department of Health include cross-training and just-in-time education for interdisciplinary collaboration. ${ }^{13}$ The education we provided may have been improved by following a model similar to that used by Hester et $\mathrm{al}^{9}$ with medical students at the University of Michigan. They provided education designed to teach RTE-specific functions and skills in collaboration with RTs. A hybrid model was developed beginning with online, skills-based modules followed by in-person skills training sessions. ${ }^{9}$ Different modules provided an array of learning checks and competency 


\section{RT Extenders' Comfort With Mechanical Ventilation}

assessments to assure that the RTEs were progressing appropriately. To conclude the training, the students were evaluated via in-person practical assessments to assure competency. ${ }^{9}$

Survey respondents reported an appropriate amount of reliance on RTs during their ICU rotations. Although the practice of respiratory therapy has been found to vary between geographic regions, the technical skills are similar (or identical). ${ }^{15}$ Some hospitals utilize RTs to the full extent of their license, while others depend on other providers for decision-making while the RTs carry out tasks. ${ }^{15}$ Still, RTs remain the clinical experts and first-line assets during times of crisis due to required competencies and their expertise in mechanical ventilation. ${ }^{3,16}$ For these reasons, hospital systems should be selective in deciding what specialty will act as RTEs during patient surges.

One area of concern reported in our survey, which related to the RTE role, was replacing RTs with providers outside the respiratory therapy profession. Replacing experienced critical care RTs, however, was never the goal. There are a number of resources that recommend supplementing critical care RTs with nonclinical staff., ${ }^{70,13}$ Project XTREME (Cross-Training Respiratory Extenders for Medical Emergencies), for example, is a disaster-preparedness guideline for providing education to nonclinical RTs and non-respiratory therapy professionals assisting in the delivery of mechanical ventilation. ${ }^{10}$ This educational model relies on interactive videos and competency validation in a laboratory setting. The authors suggest that this education can be deployed as just-in-time training. ${ }^{10} \mathrm{We}$ also followed guidance by Rubinson et al, ${ }^{7}$ who similarly suggested that RTs supervise noncritical care staff. ${ }^{10}$ Regardless, $11 \%$ of the positive feedback received was related to patient care and safety as a benefit of the RTE role in the ICU. A safety concern unrelated to the role of RTE had to do with the number of ventilators that a single RT can safely manage. ${ }^{17}$ Our experience showed that the use of RTEs adequately bolstered our staffing levels so patient safety could be prioritized. Post hoc inquiry into patient safety events revealed that there were no adverse events related to the deployment of RTEs reported in our hospitals during the COVID-19 pandemic. This could be explained by the fact that we simultaneously deployed RTs in our tele-ICU as an extra layer of protection against safety events related to COVID-19. ${ }^{18}$

Resource allocation was an important aspect of planning for the initial patient surge. ${ }^{7,8,10,13}$ As suggested in Project XTREME, ${ }^{10}$ there are 2 approaches to preparing for patient surge. First is lowering demand on the institution, and second is increasing resource supply. There are a number of approaches to accomplish this, including but not limited to discharge planning and patient diversion, as well as increasing the amount of available equipment and staff. ${ }^{10}$ Our institution stopped elective surgeries, which allowed us to utilize the CRNAs as RTEs. We are aware of several institutions, notably the University of Michigan, that utilized cross-training of other disciplines during COVID-19. In terms of resource reallocation, Indiana University Health reported successfully treating all of their COVID-19 patients without overburdening the system. ${ }^{8}$ Their plans also included "reverse surge" planning, a phase in which elective procedures and other out-patient care were resumed with renewed emphasis on early discharge, both from the ambulatory surgery center and within the hospital itself. ${ }^{8}$

The deployment of CRNAs as RTEs elucidated the idea that, as pandemics and other mass casualty events arise, as technology advances, and as new therapies are discovered, emphasis should be placed on preparedness and interdisciplinary collaboration. ${ }^{7,12}$ Subsequently, there should be significant ongoing effort and commitment to develop protocols and training guidelines for rapid deployment of staffing resources in collaboration with clinical partners including medical directors, anesthesia physician leaders, nursing leadership, and respiratory therapy leadership. ${ }^{7}$ Advanced planning for times of high patient volumes may limit the need for rapid decision-making when overwhelming situations, such as a pandemic, occur and can help organizations respond effectively and enable them to provide patients with safe, high-quality care. ${ }^{7}$ Despite this guidance from the Chest Task Force on Mass Critical Care, the COVID-19 pandemic highlighted infrastructure needs.

\section{Limitations}

Our study had several limitations. First, the survey tool used was not validated. Although reviewed by 4 of the authors prior to distribution, we did not apply principle components analysis or check for consistency. Additionally, we did not use standardized training tools or test CRNAs for competence in the use of critical care ventilators. Their level of comfort was based only on the anesthetists' subjective opinion at the time of the survey. At the same time that CRNAs were deployed, our health system added virtual RTs to our tele-ICU service, adding an extra layer of staff support which may have affected CRNA experience, and therefore, the survey results. Finally, the respondents represent a relatively small sample size. For these reasons, our results may not be generalizable to other health systems. More research is needed, with more participants, to fully understand the experience of RTEs. Future research should be inclusive of other disciplines working as RTEs so that comparisons can be made between non-respiratory therapy professionals and interprofessional comfort level and competence when using ventilators.

\section{Conclusions}

Comfort level with mechanical ventilation increased for CRNAs working alongside RTs during the COVID-19 


\section{RT Extenders' Comfort With Mechanical Ventilation}

pandemic. It is reasonable to deploy other disciplines with less experience managing critical care mechanical ventilators as RTEs. More research is needed to understand the impact of interdisciplinary collaboration on patient care and outcomes.

\section{ACKNOWLEDGMENTS}

We appreciate the hard work and dedication to patient care that was delivered by the CRNAs and RTs in the Penn Medicine Health System during the COVID-19 pandemic.

\section{REFERENCES}

1. Gattinoni L, Chiumello D, Caironi P, Busana M, Romitti F, Brazzi L, et al. COVID-19 pneumonia: different respiratory treatments for different phenotypes? Intensive Care Med 2020;46(6):1099-1102.

2. Marini JJ, Gattinoni L. Management of COVID-19 respiratory distress. JAMA 2020;323(22):2329-2330.

3. Kacmarek RM. Mechanical ventilation competencies of the respiratory therapist in 2015 and beyond. Respir Care 2013;58(6):1087-1096.

4. Spinelli A, Pellino G. COVID-19 pandemic: perspectives on an unfolding crisis. Br J Surg 2020;107(7):785-787.

5. Li R, Rivers C, Tan Q, Murray MB, Toner E, Lipsitch M. Estimated demand for US hospital inpatient and intensive care unit beds for patients with COVID-19 based on comparisons with Wuhan and Guangzhou, China. JAMA Netw Open 2020;3(5):e208297-e208297.

6. Berlin DA, Gulick RM, Martinez FJ. Severe COVID-19. N Engl J Med 2020 [Epub ahead of print] doi: CrossRef.

7. Rubinson L, Hick JL, Curtis JR, Branson RD, Burns S, Christian MD, et al. Definitive care for the critically ill during a disaster: medical resources for surge capacity: from a Task Force for Mass Critical Care Summit Meeting, Jan 26-27, 2007, Chicago, IL. Chest 2008;133(5 Suppl):32S-50S.

8. Meneghini RM. Resource re-allocation during the COVID-19 pandemic in a suburban hospital system: implications for outpatient hip and knee arthroplasty. J Arthroplasty 2020;35(7S):S15-S18.

9. Hester TB, Cartwright JD, DiGiovine DG, Karlic KJ, Kercheval JB, DiGiovine B, et al. Training and deployment of medical students as respiratory therapist extenders during COVID-19. ATS Sch 2020;1 (2): 145-151.

10. Hanley ME, Bogdan GM. Mechanical ventilation in mass casualty scenarios. Augmenting staff: project XTREME. Respir Care 2008;53 (2):176-189.

11. American Association of Nurse Anesthetists. Guidelines regarding the Role of the Certified Registered Nurse Anesthetist in Mass Casualty Incident Preparedness and Response. 2014. Available at: https://www. aana.com/docs/default-source/practice-aana-com-web-documents-(all)/ guidelines-regarding-the-role-of-the-crna-in-mass-casualty-incidentpreparedness-and-response.pdf?sfvrsn $=870049 \mathrm{~b} 1 \_4$. Accessed November 17, 2020.

12. American Association of Nurse Anesthetists. Scope of Nurse Anesthesia Practice. 2020. Available at: https://www.aana.com/docs/ default-source/practice-aana-com-web-documents-(all)/scope-of-nurseanesthesia-practice.pdf?sfvrsn=250049b1_2. Accessed November 17, 2020.

13. Pennsylvania Department of Health, Hospital and Healthsystem Association of Pennsylvania. Interim Pennsylvania Crisis Standards for Pandemic Guidelines. 2020. Available at: https://www.health.pa.gov/ topics/Documents/Diseases \%20and\%20Conditions/COVID-19\% 20Interim \%20Crisis $\% 20$ Standards $\% 20$ of $\% 20$ Care.pdf. Accessed November 17, 2020.

14. Ouersighni A, Ghazali DA. Contribution of certified registered nurse anaesthetists to the management of the COVID-19 pandemic health crisis. Intensive Crit Care Nurs 2020;60:102888.

15. Armaghan R, Geesey B, Juby J, Amador-Castaneda J, Bollinger A, Roberts KJ, et al. Practice of respiratory therapists in Pennsylvania: a statewide survey. Respir Care 2020;65(7):972-976.

16. Barnes TA, Gale DD, Kacmarek RM, Kageler WV. Competencies needed by graduate respiratory therapists in 2015 and beyond. Respir Care 2010;55(5):601-616.

17. Parker AM, Liu X, Harris AD, Shanholtz CB, Smith RL, Hess DR, et al. Respiratory therapy organizational changes are associated with increased respiratory care utilization. Respir Care 2013;58(3):438-449.

18. Pierce M, Gudowski SW, Roberts KJ, Scott MJ, Laudanski K. Establishing a telemedicine respiratory service (eRT) in the COVID-19 Pandemic. J Cardiothorac Vasc Anesth 2020;10.1053/ j.jvca.2020.11.021 [Epub ahead of print].

This article is approved for Continuing Respiratory Care Education credit. For information and to obtain your CRCE

(free to AARC members) visit www.rcjournal.com 